\title{
Effectiveness of interventions to increase uptake and completion of treatment for diabetic retinopathy in low- and middle- income countries: a rapid review protocol
}

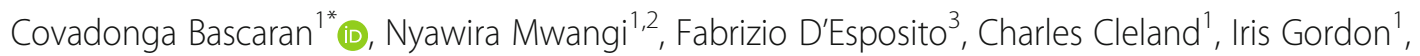
Juan Alberto Lopez Ulloa ${ }^{4}$, Ranad Maswadi ${ }^{5}$, Shaffi Mdala ${ }^{6}$, Jacqueline Ramke ${ }^{1,7}$, Jennifer R. Evans ${ }^{1}$ and Matthew Burton ${ }^{1,8}$

\begin{abstract}
Background: Vision loss due to diabetic retinopathy can largely be prevented or delayed through treatment. Patients with vision-threatening diabetic retinopathy are typically offered laser or intravitreal injections which often require more than one treatment cycle. However, treatment is not always initiated, or it is not completed, resulting in poor visual outcomes. Interventions aimed at improving the uptake or completion of treatment for diabetic retinopathy can potentially help prevent or delay visual loss in people with diabetes.

Methods: We will search MEDLINE, Embase, Global Health and Cochrane Register of Studies for studies reporting interventions to improve the uptake of treatment for diabetic retinopathy (DR) and/or diabetic macular oedema (DMO), compared with usual care, in adults with diabetes. The review will include studies published in the last 20 years in the English language. We will include any study design that measured any of the following outcomes in relation to treatment uptake and completion for DR and/or DMO: (1) proportion of patients initiating treatment for DR and/or DMO among those to whom it is recommended, (2) proportion of patients completing treatment for DR and/or DMO among those to whom it is recommended, (3) proportion of patients completing treatment for DR and/or DMO among those initiating treatment and (4) number and proportion of DR and/or DMO rounds of treatment completed per patient, as dictated by the treatment protocol. For included studies, we will also report any measures of cost-effectiveness when available. Two reviewers will screen search results independently. Risk of bias assessment will be done by two reviewers, and data extraction will be done by one reviewer with verification of $10 \%$ of the papers by a second reviewer. The results will be synthesised narratively.
\end{abstract}

(Continued on next page)

* Correspondence: Covadonga.bascaran@lshtm.ac.uk

'London School of Hygiene \& Tropical Medicine, Keppel Street, London

WC1E 7HT, UK

Full list of author information is available at the end of the article

(c) The Author(s). 2021 Open Access This article is licensed under a Creative Commons Attribution 4.0 International License, which permits use, sharing, adaptation, distribution and reproduction in any medium or format, as long as you give appropriate credit to the original author(s) and the source, provide a link to the Creative Commons licence, and indicate if changes were made. The images or other third party material in this article are included in the article's Creative Commons licence, unless indicated otherwise in a credit line to the material. If material is not included in the article's Creative Commons licence and your intended use is not permitted by statutory regulation or exceeds the permitted use, you will need to obtain permission directly from the copyright holder. To view a copy of this licence, visit http://creativecommons.org/licenses/by/4.0/. The Creative Commons Public Domain Dedication waiver (http://creativecommons.org/publicdomain/zero/1.0/) applies to the data made available in this article, unless otherwise stated in a credit line to the data. 


\begin{abstract}
(Continued from previous page)
Discussion: This rapid review aims to identify and synthesise the peer-reviewed literature on the effectiveness of interventions to increase uptake and completion of treatment for DR and/or DMO in LMICs. The rapid review methodology was chosen in order to rapidly synthesise the available evidence to support programme implementers and policy-makers in designing evidence-based health programmes and public health policy and inform the allocation of resources.
\end{abstract}

Systematic review registration: OSF osf.io/h5wgr

Keywords: Low- and middle-income countries, Diabetic retinopathy, Patient acceptance of health care, Treatment outcome

\section{Background}

Vision loss due to diabetic retinopathy (DR) can largely be prevented or delayed through treatment. DR programmes focus on regular screening of people living with diabetes and referral to eye care services for further assessment. Patients with vision-threatening diabetic retinopathy (VTDR), including proliferative diabetic retinopathy (PDR) and/or diabetic macular oedema (DMO), are then offered laser treatment and/or intravitreal injections which often require more than one treatment visit $[1,2]$. However, the effectiveness of DR treatment is dependent on the timely initiation and completion of the prescribed treatment course.

Different factors-both from the demand and the supply side-can affect treatment uptake and completion [3]. Health systems in low- and middle-income country (LMIC) settings experience limitations in the delivery of high-quality and timely DR treatment, including low numbers of ophthalmologists trained in DR management, inadequate referral systems, limited access to technology and equipment and the lack of health information systems and policies that address DR management $[4,5]$. LMIC populations have competing health needs that determine their priorities and health-seeking behaviours in relation to eye health. Barriers like fear, price of services, waiting times or distance can lead to patients missing treatment, or initiating treatment but not completing it, resulting in poor visual outcomes. Low uptake of DR treatment compounds the challenge to prevent visual loss in people with diabetes and wastes resources in already limited health systems [6]. Interventions aiming to improve uptake and completion of DR treatment need to target the relevant factors in the healthcare environment in which the patients are to receive treatment [6].

Access to DR services is a widespread problem in LMICs. Studies of DR services in Pakistan, Oman and China reported that $29.5 \%, 22.8 \%$ and $27.9 \%$ of patients respectively did not initiate treatment [7-9]. In the study in China, an additional $17.6 \%$ did not complete the treatment course, for reasons including being unaware of the importance of treatment and the need to complete the full course, and fear of treatment [9]. In high-income countries, the proportion of patients not completing treatment tends to be lower than in low-income countries but still significant. A review in the USA reported that approximately $15 \%$ of patients did not initiate treatment and 15\% of those did not complete it [10].

Laser photocoagulation is a highly effective treatment for PDR and DMO [11-13]. DMO and PDR can be treated with anti-vascular endothelial growth factor (VEGF) via intravitreal injections [14, 15]. Intravitreal steroids, while not indicated as first-line therapy for most eyes with DMO, have been used by some clinicians for eyes that do not adequately respond to anti-VEGF therapy $[16,17]$. Anti-VEGF is also indicated in agerelated macular degeneration (ARMD) [18]. However, there is evidence that the uptake of anti-VEGF by patients with DMO is significantly lower compared to patients with ARMD [19-21]. People with diabetes often have other co-morbidities that increase their need to attend hospital appointments, so completing an antiVEGF treatment regime can be a significant additional burden. Patients receiving intravitreal injections report high levels of anxiety and significant psychological impact [22]. The dependence on a caregiver to accompany the patient and the financial cost of treatment also pose a burden on the patient [23, 24].

This rapid review aims to identify and summarise the most recent information on the effectiveness of interventions to increase uptake and completion of treatment for DR and/or DMO in LMICs.

\section{Methods \\ Rapid review question}

What interventions are effective in increasing uptake and completion of treatment for DR and/or DMO among people with diabetes in LMICs?

\section{Protocol and registration}

We used the STARR Decision Tool and the WHO practical guide to carefully consider the methods most appropriate for this rapid review and the possible limitations introduced by those methods $[25,26]$. 
Additional file 1 provides the adapted Preferred Reporting Items for Systematic Reviews and Metaanalysis Protocols (PRISMA-P) which is used to report this protocol.

The protocol was registered on the Open Science Framework (OSF): osf.io/h5wgr; more details are provided in Additional file 2. Any protocol amendments will be documented in the registration site [27].

\section{Eligibility criteria}

We will limit the search to peer-reviewed publications of studies conducted in LMICs in any setting in the last 20 years (i.e. community or facility based), which examine the uptake and/or completion of treatment prescribed for DR or DMO. We defined LMICs according to the World Bank Classification for 2019. Studies will only be included if they report at least one of the first four outcomes. We will restrict to publications in the English language. We will only include studies where a full-text report is available (Table 1$)$.

\section{Information sources}

We will search MEDLINE, Embase, Global Health and the Cochrane Register of Studies. The search strategies will be developed by a Cochrane Eyes and Vision Information Specialist (IG). We will search the reference lists of included studies. Grey literature will not be considered.

\section{Search strategy}

The search strategy is included in Additional file 3.

\section{Data management and selection process}

Screening will be done using online review management software (Covidence, Veritas Health Innovation, Melbourne, Australia; available at www.covidence.org). Each title and abstract will be screened independently by two reviewers. Disagreements will be resolved by discussion. Full-text articles describing potentially relevant studies will be screened by two reviewers independently against the inclusion and exclusion criteria and any differences resolved by consensus. A
PRISMA flow diagram will be completed to summarise the study selection process.

\section{Risk of bias assessment}

Risk of bias will be assessed by two reviewers independently using SIGN critical appraisal checklists for this purpose, available at https://www.sign.ac.uk. Any differences will be agreed by consensus, and a third researcher will be consulted if any differences are unresolved. The overall risk of bias for included studies will be reported in narrative form and used to interpret the findings of the review.

\section{Data extraction}

A custom-designed Excel data extraction form will be piloted by two reviewers on 3 papers and adapted accordingly. Data will be extracted by one reviewer, and the accuracy of data extraction will be checked by a second reviewer for $10 \%$ of studies. If there are significant data extraction errors (for example, important errors in more than $1 \%$ of records), then a further set of records will be checked. The following data items will be collected for each identified intervention (Table 2).

\section{Data synthesis}

We anticipate that there will be clinical and methodological diversity in the studies that we find, so we plan to summarise the data narratively, following SWiM reporting guidance: Synthesis Without Meta-analysis [28].

We will report outcomes in terms of proportions in intervention and comparator groups and will calculate the risk ratio with $95 \%$ confidence intervals as the main measure of effect, but this will be dependent on how the data are reported by the included studies. In observational studies, for example, adjusted odds ratios may be the most appropriate measure of effect. We will present key characteristics such as study design, sample size and risk of bias in tables and use visual displays for effect estimates when possible. We will consider heterogeneity by examining study design, geographical location, demographic characteristics such as age and sex as well as the nature of the interventions and the settings in which

Table 1 Eligibility criteria

\begin{tabular}{|c|c|}
\hline Population & Adults (aged $\geq 18$ years) with type 1 or type 2 diabetes in LMICs who have been identified as needing treatment for DR and/or DMO \\
\hline Intervention & Any intervention that seeks to increase uptake and/or completion of treatment for DR and/or DMO among adults with diabetes \\
\hline Comparator & Usual care or another intervention seeking to increase uptake and/or completion of treatment for DR and/or DMO \\
\hline Outcomes & $\begin{array}{l}\text { 1. Proportion of patients initiating treatment for } \mathrm{DR} \text { and/or } \mathrm{DMO} \text { among those to whom it is recommended } \\
\text { 2. Proportion of patients completing treatment for } \mathrm{DR} \text { and/or } \mathrm{DMO} \text { among those to whom it is recommended } \\
\text { 3. Proportion of patients completing treatment for } \mathrm{DR} \text { and/or DMO among those initiating treatment } \\
\text { 4. Number and proportion of DR and/or DMO rounds of treatment completed per patient, as dictated by the treatment protocol } \\
\text { 5. Cost-effectiveness of intervention to increase DR/DMO treatment uptake or completion }\end{array}$ \\
\hline Study design & Any interventional or observational comparative study \\
\hline
\end{tabular}


Table 2 Data items

\begin{tabular}{ll}
\hline Data categories & Example data outcomes \\
\hline $\begin{array}{l}\text { Publication } \\
\text { characteristics }\end{array}$ & Authors, title, publication date, journal citation \\
Study characteristics & Design \\
& Dates of data collection \\
Population & Country \\
characteristics & Population demographics (age, gender) \\
& Sample size \\
& Setting (rural/urban, community/hospital) \\
Intervention & Intervention target: patients, providers, policy-makers \\
characteristics & Treatment indication: pan retinal laser photocoagulation (PRP) for PDR, focal laser, intravitreal anti-VEGF or steroids for DMO \\
& Treatment completion guideline, e.g. number of laser sessions, number of burns and number of injections \\
& Type of intervention, e.g. guidelines, treatment regime, educational programmes, financial subsidies, follow-up prompts and \\
& reminders, ongoing quality assurance and improvement processes, electronic patient management \\
& Factors associated with uptake/completion of treatment: provider and patient \\
& Proportion of patients initiating treatment for DR and/or DMO among those to whom it is recommended \\
& Proportion of patients completing treatment for DR and/or DMO among those to whom it is recommended \\
& Proportion of patients completing treatment for DR and/or DMO among those initiating treatment \\
& Number and proportion of DR and/or DMO rounds from the recommended treatment protocol completed per patient \\
& Cost-effectiveness of intervention to increase DR/DMO treatment uptake or completion \\
\hline
\end{tabular}

they have been applied. If sufficient data are available, we will calculate $I^{2}$ which is a measure of the percentage of variation across studies that is due to heterogeneity rather than chance. We will use the GRADE approach, as applied to narrative syntheses, to assess the certainty of the synthesis findings [29].

Depending on the findings, we will group studies by setting, intervention target (patients, health providers, health system), type of intervention, study design and outcomes. For each comparison and outcome, we will provide a description of the findings alongside the certainty of the evidence, ensuring consistency with the review question and providing a judgement as to the extent to which the studies contribute to the synthesis.

\section{Discussion}

This rapid review aims to identify and synthesise the peer-reviewed literature on the effectiveness of interventions to increase uptake and completion of treatment for DR and/or DMO in LMICs. The rapid review methodology was chosen in order to rapidly synthesise the available evidence to support programme implementers and policy-makers in designing evidence-based health programmes and public health policy and inform the allocation of resources. This review may also identify gaps in the evidence that could inform further research priorities related to the management of diabetic retinopathy in LMICs.

\section{Supplementary Information}

The online version contains supplementary material available at https://doi. org/10.1186/s13643-020-01562-9.

Additional file 1:. Reporting standards - PRISMA-P Checklist.

Additional file 2:. Registration OSF: https://osf.io/h5wgr/

Additional file 3:. Search strategy.

\section{Abbreviations}

DR: Diabetic retinopathy; DMO: Diabetic macular oedema; LMIC: Low- and middle-income country; VTDR: Vision-threatening diabetic retinopathy; OSF: Open science framework; PDR: Proliferative diabetic retinopathy; VEGH: Vascular endothelial growth factor; ARMD: Age-related macular degeneration; PRISMA-P: Preferred reporting items for systematic reviews and meta-analysis protocols; SING: Scottish intercollegiate guidelines network; PRP: Pan retinal laser photocoagulation; SWiM: Synthesis without meta-analysis; GRADE: Grading of recommendations assessment, development and evaluation

\section{Acknowledgements}

Not applicable.

\section{Authors' contributions}

FD conceived the idea for the review. CB and NM drafted and revised the protocol with suggestions from JE, JR, FD, MJB, CC, RM, SM, and JALU, who reviewed the protocol and provided feedback on the draft. IG constructed the search. The authors read and approved the final manuscript.

\section{Funding}

This review was commissioned and funded by Fred Hollows Foundation (FHF) Australia. MJB is supported by the Wellcome Trust (207472/Z/17/Z).

Availability of data and materials

Not applicable—no data sets currently available. 
Ethics approval and consent to participate

Not applicable.

\section{Consent for publication}

Not applicable.

\section{Competing interests}

None declared.

\section{Author details}

'London School of Hygiene \& Tropical Medicine, Keppel Street, London WC1E 7HT, UK. ${ }^{2}$ Kenya Medical Training College, Nairobi, Kenya. ${ }^{3}$ The Fred Hollows Foundation, Melbourne, Australia. ${ }^{4}$ Centro Mexicano de Salud Visual Preventiva, Mexico DF, Mexico. ${ }^{5}$ St Thomas's Hospital, Westminster Bridge Road, London SE1 7EH, UK. ${ }^{6}$ Queen Elizabeth Central Hospital, P.O.Box 95, Blantyre, Malawi. ${ }^{7}$ School of Optometry and Vision Science, University of Auckland, Auckland, New Zealand. ${ }^{8}$ Moorfields Eye Hospital, London, UK.

Received: 14 May 2020 Accepted: 17 December 2020

Published online: 14 January 2021

\section{References}

1. Wong TY, Sun J, Kawasaki R, Ruamviboonsuk P, Gupta N, Lansingh VC, et al. Guidelines on diabetic eye care: the International Council of Ophthalmology recommendations for screening, follow-up, referral, and treatment based on resource settings. Ophthalmology. 2018 Oct;125(10):1608-22.

2. Mwangi N. ea. Clinical guidelines for diabetic retinopathy in Kenya: an executive summary of the recommendations. JOECSA. 2017;21(2):33-9.

3. Jacobs B, Ir P, Bigdeli M, Annear PL, Van Damme W. Addressing access barriers to health services: an analytical framework for selecting appropriate interventions in low-income Asian countries. Health Policy Plann. 2012;27(4): 288-300.

4. Poore S, Foster A, Zondervan M, Blanchet K. Planning and developing services for diabetic retinopathy in sub-Saharan Africa. Int J Health Policy Manage. 2015;4(1):19-28

5. Burgess Pl, Msukwa G, Beare NA. Diabetic retinopathy in sub-Saharan Africa: meeting the challenges of an emerging epidemic. BMC Med. 2013;11:157.

6. De Geest S, Sabaté E. Adherence to long-term therapies: evidence for action. Eur J Cardiovasc Nurs. 2003;2(4):323. https://doi.org/10.1016/S14745151(03)00091-4

7. Memon S, Ahsan S, Alvi R, Fawwad A, Basit A, Shera S, et al. Retinal screening acceptance, laser treatment uptake and follow-up response in diabetics requiring laser therapy in an urban diabetes care centre. J Coll Phys Surg Pak. 2015;25(10):743-6.

8. Khandekar R, Al Lawati J, Barakat N. A retrieval system for patients with avoidable blindness due to diabetic retinopathy who do not present for ophthalmic assessment in Oman. Middle East Afr J Ophthalmol. 2011;18(2): 93-7.

9. Hua W, Cao S, Cui J, Maberley D, Matsubara J. Analysis of reasons for noncompliance with laser treatment in patients of diabetic retinopathy. Can J Ophthalmol. 2017;52(Suppl 1):S34-s8.

10. Will JC, German RR, Schuman E, Michael S, Kurth DM, Deeb L. Patient adherence to guidelines for diabetes eye care: results from the diabetic eye disease follow-up study. Am J Public Health. 1994;84(10):1669-71.

11. Group TDRSR. Photocoagulation for diabetic macular edema. early treatment diabetic retinopathy study report number 1. Early treatment diabetic retinopathy study research group. Arch Ophthalmol. 1985;103(12): 1796-806.

12. Jorge EC, Jorge EN, Botelho M, Farat JG, Virgili G, El Dib R. Monotherapy laser photocoagulation for diabetic macular oedema. Cochrane Database Syst Rev. 2018;10:Cd010859

13. Moutray T, Evans JR, Lois N, Armstrong DJ, Peto T, Azuara-Blanco A. Different lasers and techniques for proliferative diabetic retinopathy. Cochrane Database Syst Rev. 2018;3:Cd012314

14. Martinez-Zapata MJ, Marti-Carvajal AJ, Sola I, Pijoan JI, Buil-Calvo JA, Cordero $\mathrm{JA}$, et al. Anti-vascular endothelial growth factor for proliferative diabetic retinopathy. Cochrane Database Syst Rev. 2014 Nov 24(11):Cd008721.

15. Virgili G, Parravano M, Evans JR, Gordon I, Lucenteforte E. Anti-vascular endothelial growth factor for diabetic macular oedema: a network metaanalysis. Cochrane Database Syst Rev. 2018;10:Cd007419.
16. Grover D, Li TJ, Chong CC. Intravitreal steroids for macular edema in diabetes. Cochrane Database Syst Rev. 2008;23(1):Cd005656.

17. Mehta H, Hennings C, Gillies MC, Nguyen V, Campain A, Fraser-Bell S. Antivascular endothelial growth factor combined with intravitreal steroids for diabetic macular oedema. Cochrane Database Syst Rev. 2018;4:Cd011599.

18. Solomon SD, Lindsley K, Vedula SS, Krzystolik MG, Hawkins BS. Anti-vascular endothelial growth factor for neovascular age-related macular degeneration. Cochrane Database Syst Rev. 2019;3:Cd005139.

19. Angermann R, Rauchegger T, Nowosielski Y, Casazza M, Bilgeri A, Ulmer $H_{\text {, }}$ et al. Treatment compliance and adherence among patients with diabetic retinopathy and age-related macular degeneration treated by anti-vascular endothelial growth factor under universal health coverage. Graefes Arch Clin Exp Ophthalmol. 2019;257(10):2119-25.

20. Jansen ME, Krambeer CJ, Kermany DS, Waters JN, Tie W, Bahadorani S, et al. Appointment compliance in patients with diabetic macular edema and exudative macular degeneration. Ophthalmic Surg Lasers Imaging Retina. 2018:49(3):186-90.

21. Best AL, Fajnkuchen F, Nghiem-Buffet S, Grenet T, Quentel G, DelahayeMazza C, et al. Treatment efficacy and compliance in patients with diabetic macular edema treated with ranibizumab in a real-life setting. $J$ Ophthalmol. 2018;2018:4610129.

22. Spooner KL, Guinan G, Koller S, Hong T, Chang AA. Burden of treatment among patients undergoing intravitreal injections for diabetic macular oedema in Australia. Diab Metab Syndr Obes Targets Ther. 2019;12:1913-21.

23. Gohil R, Crosby-Nwaobi R, Forbes A, Burton B, Hykin P, Sivaprasad S. Caregiver burden in patients receiving ranibizumab therapy for neovascular age related macular degeneration. PloS one. 2015;10(6):e0129361.

24. Hanemoto T, Hikichi Y, Kikuchi N, Kozawa T. The impact of different antivascular endothelial growth factor treatment regimens on reducing burden for caregivers and patients with wet age-related macular degeneration in a single-center real-world Japanese setting. PloS one. 2017;12(12):e0189035.

25. Abdullah P, Eva K, Katherine C, Marrissa M-SJ, Ruth W. SelecTing Approaches for Rapid Reviews (STARR) Decision Tool 2019.

26. Andrea C. Tricco EVL, Sharon E. Straus. Rapid reviews to strengthen health policy and systems: a practical guide. In: WHO, editor. 2017.

27. Bascaran C, Mwangi N, D'Esposito F, Cleland CR, Ulloa JAL, Gordon I, Burton $M$. Effectiveness of interventions to increase uptake and completion of treatment for diabetic retinopathy in low- and middle-income countries: a rapid review protocol. Retrieved from https://doi.org/10.17605/OSF.IO/ H5WGR.

28. Campbell M, McKenzie JE, Sowden A, Katikireddi SV, Brennan SE, Ellis $\mathrm{S}$, et al. Synthesis without meta-analysis (SWiM) in systematic reviews: reporting guideline. BMJ. 2020;368:16890

29. Murad MH, Mustafa RA, Schunemann HJ, Sultan S, Santesso N. Rating the certainty in evidence in the absence of a single estimate of effect. Evidence-based medicine. 2017 Jun:22(3):85-7.

\section{Publisher's Note}

Springer Nature remains neutral with regard to jurisdictional claims in published maps and institutional affiliations.

Ready to submit your research? Choose BMC and benefit from:

- fast, convenient online submission

- thorough peer review by experienced researchers in your field

- rapid publication on acceptance

- support for research data, including large and complex data types

- gold Open Access which fosters wider collaboration and increased citations

- maximum visibility for your research: over $100 \mathrm{M}$ website views per year

At BMC, research is always in progress.

Learn more biomedcentral.com/submission 